\title{
High prevalence of Klebsiella pneumoniae carbapenemase-mediated resistance in K. pneumoniae isolates from Egypt
}

L. Metwally, ${ }^{1}$ N. Gomaa, ${ }^{1}$ M. Attallah ${ }^{2}$ and N. Kamel ${ }^{3}$

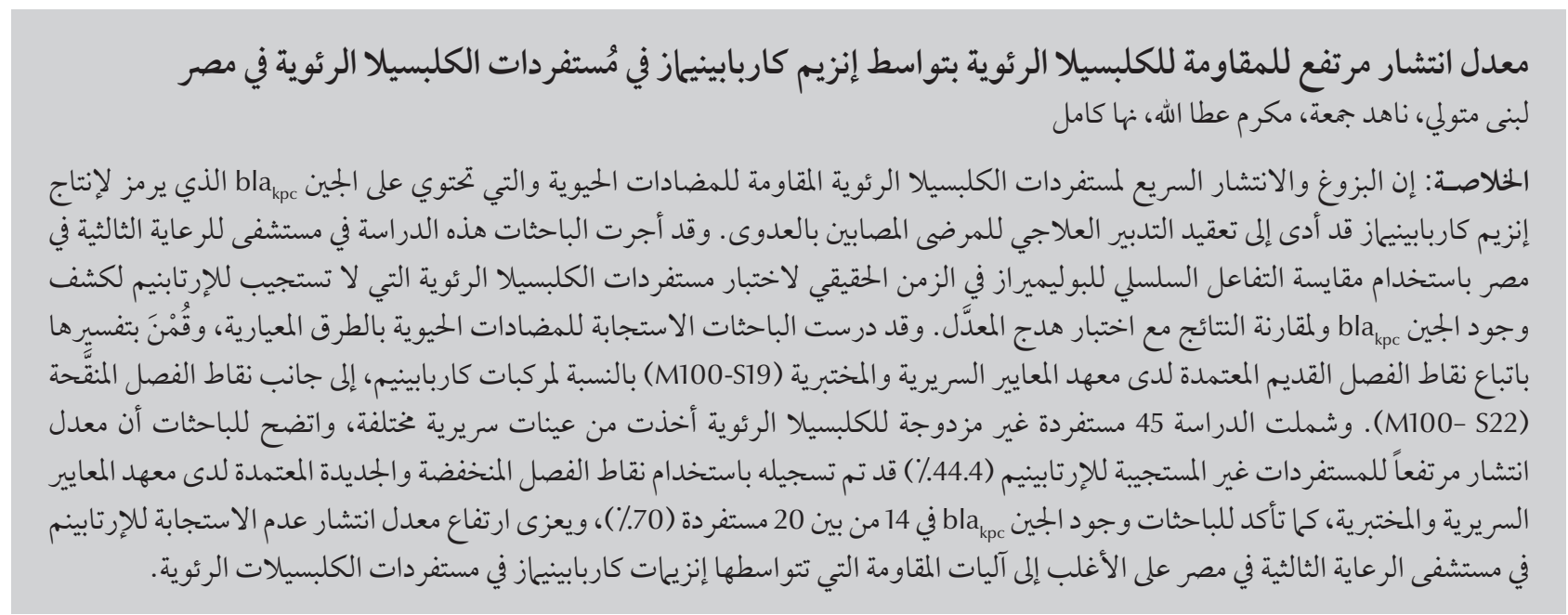

ABSTRACT The emergence and rapid spread of antibiotic-resistant Klebsiella pneumoniae isolates harbouring the $b_{\text {KPC }}$ gene that encodes for carbapenemase production have complicated the management of patient infections. This study in a tertiary care hospital in Egypt used real-time PCR assay to test ertapenem-nonsusceptible isolates of K. pneumoniae for the presence of the $b l a_{\mathrm{KPC}}$ gene and compared the results with modified Hodge test. Antibiotic sensitivity was performed by standard methods, and interpreted following both the old CLSI breakpoints (M100-S19) for carbapenems and the revised breakpoints (M100-S22). From the 45 non-duplicate isolates of $K$. pneumoniae recovered from different clinical specimens, a high prevalence of ertapenem-nonsusceptible isolates (44.4\%) was reported using the new lower CLSI breakpoints. The bla $a_{\mathrm{KPC}}$ gene was confirmed in 14/20 (70.0\%) of these isolates. The high prevalence of ertapenem nonsusceptibility at a tertiary care hospital in Egypt was predominantly attributed to K. pneumoniae carbapenemase-mediated resistance mechanisms in K. pneumoniae isolates.

Prévalence élevée de la résistance de Klebsiella pneumoniae médiée par les carbapénèmases dans des isolats de K. pneumoniae en Égypte

RÉSUMÉ L'émergence et la propagation rapide des souches de Klebsiella pneumoniae résistantes aux antibiotiques et porteuses du gène $b l a_{\mathrm{KPC}}$ codant la production de carbapénèmases ont compliqué la prise en charge des infections des patients. La présente étude menée dans un hôpital de soins tertiaires en Égypte a utilisé la méthode de PCR en temps réel pour évaluer la présence du gène $b l a_{\mathrm{KPC}}$ dans les isolats de $K$. pneumoniae non sensibles à l'ertapénème, puis a comparé les résultats à l'aide du test de Hodge modifié. La sensibilité aux antibiotiques a été évaluée à l'aide des méthodes standards, puis a été interprétée selon les anciens seuils du Clinical and Laboratory Standards Institute (M100-S19) pour les carbapénèmes et selon les seuils révisés (M100-S22). Après I'analyse des 45 isolats non-dupliqués de $K$. pneumoniae prélevés à partir de différents échantillons cliniques, une prévalence élevée d'isolats non sensibles à l'ertapénème (44,4 \%) a été rapportée selon les nouveaux seuils plus bas du Clinical and Laboratory Standards Institute. La présence du gène bla $_{\mathrm{KPC}}$ a été confirmée dans 14 isolats sur 20 (70,0 \%). La forte prévalence de la non sensibilité à l'ertapénème dans un hôpital de soins tertiaires en Égypte était principalement imputable aux mécanismes de résistance médiés par les carbapénèmases dans les isolats de K. pneumoniae.

${ }^{7}$ Department of Microbiology; ${ }^{3}$ Department of Clinical Pathology, Faculty of Medicine, Suez Canal University, Ismailia, Egypt (Correspondence to L. Metwally: Lobna.metwally@gmail.com).2Department of Microbiology, Faculty of Medicine, Ain Shams University, Cairo, Egypt.

Received: 28/08/12; accepted: 17/10/12 


\section{Introduction}

Klebsiella pneumoniae carbapenemases (KPCs) are Ambler class A plasmidencoded enzymes that are capable of hydrolyzing all beta-lactam antibiotics, including monobactams, extendedspectrum cephalosporins and carbapenems $[1,2]$. Originally described in 2001 [3], pathogens harbouring these antibiotic-resistance enzymes have been reported from the United States of America (USA) [4-7], France, China, Sweden, Norway, Colombia, Brazil, Scotland, Germany and Spain [8-11]. Epidemic situations have also been reported in Israel and Greece $[12,13]$. An important challenge to developing a standardized definition of bacterial isolates resistant to carbapenems is a recent (mid-2010) change in the Clinical and Laboratory Standards Institute (CLSI) interpretative criteria (breakpoints) for determining susceptibility to carbapenems among Enterobacteriaceae $[14,15]$. These new recommendations lowered the breakpoints and removed the requirement for testing for carbapenemases, e.g. by modified Hodge test (MHT), to determine susceptibility. However, based on clinical and microbiological data, ertapenem breakpoints were modified again in January 2012 (M100-S22) by doubling the dilution (to $\leq 0.5 \mu \mathrm{g} / \mathrm{mL}$ ) [16].

In addition to beta-lactam/carbapenem resistance, nonsusceptible organisms can carry genes that confer high levels of resistance to many other antimicrobials, often leaving very limited therapeutic options $[17,18]$. The $b l a_{\mathrm{KPC}}$ gene encodes for KPC enzyme production. Although carbapenemases have been identified in many species of Enterobacteriaceae, K. pneumoniae remains the most common organism carrying resistance-encoding genes [2]. Carbapenem resistance in K. pneumoniae may also be due to production of other carbapenemases [19] or to changes in outer membrane porin proteins [20], often combined with production of an extended-spectrum beta-lactamase, AmpC or both [19,21].

Molecular detection of the $b l_{\mathrm{KPC}}$ gene by polymerase chain reaction (PCR) assay provides laboratories with a means to quickly identify the presence of this important resistance determinant $[22,23]$. Considering the demonstrated potential for rapid horizontal and vertical transmission of the $b l a_{\mathrm{KPC}}$ gene, prompt recognition is important to controlling the spread of KPCs. In the present study we describe a real-time PCR assay to detect all variants of the $b a_{\mathrm{KPC}}$ gene and the use of this assay to test clinical isolates of $K$. pneumoniae. We also tested ertapenemnonsusceptible isolates using MHT.

\section{Methods}

\section{Study isolates}

A prospective study was conducted over a period of 6 months (June 2011 to December 2011) at the Suez Canal University hospital, Ismailia, Egypt. A total of 45, single-patient K. pneumoniae isolates were included in the study. These isolates were recovered from urine $(n=13)$, blood $(n=8)$, respiratory tract $(n=12)$ and other clinical sites $(n=12)$ from patients admitted to the intensive care unit and different wards of the hospital. Full identification was carried out using the API 20E system (bioMérieux).

Ethical approval to perform the study was obtained from the ethics committee in the Faculty of Medicine, Suez Canal University and the management board of the hospital. All the included patients consented to the collection of specimens before the study was initiated.

\section{Susceptibility testing}

Antibiotic susceptibility testing was determined using the modified KirbyBauer method following the CLSI guidelines. The following antimicrobial agents were included in the panel: ampicillin, amoxicillin/clavulanic acid, ceftriaxone, cefepime, cefazolin, cefoxitin, ciprofloxacin, gentamicin, tobramycin, imipenem, ertapenem, meropenem, trimethoprim/sulfamethoxazole, piperacillin, piperacillin/tazobactam and tobramycin (Oxoid).

Isolates were further subjected to minimum inhibitory concentration (MIC) testing for imipenem and meropenem using the Oxoid MIC evaluator strip (Thermo Fisher Scientific) and for ertapenem using the gradient strip Etest (bioMérieux); boxes were allowed to equilibrate at room temperature for at least $1 \mathrm{~h}$ before opening. For all isolates the inocula for strip tests were matched to a $0.5 \mathrm{McF}$ arland standard. Results were read in accordance with the manufacturers' directions and interpreted following both the old CLSI M100-S19 breakpoints and the revised breakpoints in the M100-S22 document issued in January 2012 [14-16].

Suspension of a known KPC-producing isolate [K. pneumoniae American Type Culture Collection (ATCC) BAA-1705], recovered on the MacConkey agar, was used as quality control strain. A second carbapenem-susceptible K pneumoniae (ATCC 700603) was used as negative control.

Stocks of 20 distinct single-patient K. pneumoniae isolates representing different antibiogram patterns and showing MIC $\geq 1 \mu \mathrm{g} / \mathrm{mL}(n=20)$ for ertapenem, using the revised carbapenem breakpoints (M100-S22, January 2010), were tested for carbapenemases by MHT and stored in tryptic soy broth with $20 \%$ glycerol at $-20^{\circ} \mathrm{C}$ until further testing by lla $_{\mathrm{KPC}}$ real-time PCR.

\section{Detection of $b l a_{\mathrm{KPC}}$ by real- time PCR}

Fresh, well-isolated test colonies grown on sheep-blood agar plates following overnight incubation were used for DNA extraction using the QIAamp DNA mini kit (Qiagen) according to the manufacturer's protocol. Briefly, a $2.0 \mathrm{McF}$ arland standard bacterial 
suspension was prepared in saline, and bacterial DNA was extracted from 200 $\mu \mathrm{L}\left(1.2 \times 10^{8}\right.$ colony forming units $)$ of the suspension. Extracted bacterial DNA was eluted from the columns in $100 \mu \mathrm{L}$ elution buffer and stored at $-20^{\circ} \mathrm{C}$.

The TaqMan real-time KPC PCR assay uses previously published primers and probes which detect all currently described KPC variants [24]. The sequences were as follows: for the KPC forward primer, 5'-GCG GAA CCA TTC GCT AAA CTC GAA-3'; for the $\mathrm{KPC}$ reverse primer, 5'-AGA AAG CCC TTG AAT GAG CTG CAC-3'; and for the KPC probe, 5'-/6-FAM/ ATA CCG GCT CAG GCG CAA CTG TAA GTT A/6-TAMRA/-3' (where 6-FAM represents 6-carboxyfluorescein and 6-TAMRA represents 6-carboxytetramethylrhodamine).

Real-time PCR was performed with $2 \mu \mathrm{L}$ template DNA in a total reaction volume of $10 \mu \mathrm{L}$ containing 1× LightCycler FastStart DNA master hybridization probe reagent (Roche Diagnostics), $3.5 \mathrm{mM} \mathrm{MgCl}_{2}$, and $2 \mu \mathrm{M}$ of primers for $b l a_{\mathrm{KPC}}$ and the TaqMan probe.

A negative control consisting of the reaction mixture and water (in place of template DNA) was added in each run. In addition to negative controls, a reference $K$ pneumoniae strain (ATCC BAA-1705) was selected as the positive control.

The LightCycler 2.0 instrument (Roche Diagnostics) was used for the amplification and detection of the $b l a_{\mathrm{KPC}}$ gene using the following PCR cycling conditions; after an initial denaturation step of 3 min at $95^{\circ} \mathrm{C}$, a 2-step PCR procedure was used consisting of $30 \mathrm{~s}$ at $95^{\circ} \mathrm{C}$ and 1 min at $60^{\circ} \mathrm{C}$ for 45 cycles.

Data were obtained during the annealing period. Fluorescence was measured once every cycle immediately after the $60{ }^{\circ} \mathrm{C}$ incubation (extension step). Fluorescence curves were analysed with the LightCycler software, version 4.0. The results were expressed by determination of the threshold cycle $(\mathrm{Ct})$ value which marked the cycle at which the fluorescence of the sample became significantly different from the baseline signal. A sample was regarded as positive when the LightCycler software determined a $\mathrm{Ct}$ in the quantification analysis screen.

When analysing the results, it is important to only consider amplification between $10-35$ cycles as positive. Amplification prior to 10 cycles means the template should be diluted before repeating. Amplification after 35 cycles can indicate trace contamination. The no template (water) control should not yield a product $(\mathrm{Ct}>40)$. PCR positive isolates with reduced ertapenem MIC were considered to be KPC positive.

\section{Detection of KPC by the MHT}

Isolates that were nonsusceptible to ertapenem (i.e. resistant and intermediate isolates) were also tested by the MHT previously described [25]. Briefly, a 0.5 McFarland suspension of Escherichia coli (ATCC 25922), was used to prepare a lawn culture on a Mueller-Hinton agar plate (Becton Dickinson), and a $10 \mu \mathrm{g}$ ertapenem susceptibility disk (Oxoid) was placed in the centre of the test area. Test isolates were subcultured onto sheep-blood agar plates (Becton Dickinson) to establish pure cultures. The isolate was then streaked in a straight line from the edge of the disk to the edge of the plate and was incubated overnight at $35^{\circ} \mathrm{C}$ in ambient air. After 24 hours of incubation, the plate was examined for a cloverleaf-shaped indentation at the intersection of the test organism and the E. coli ATCC 25922 within the zone of inhibition. The presence of a cloverleaf-shaped indentation was considered MHT positive.

\section{Results}

By using current breakpoints (M100S22) for carbapenem interpretation, 20 out of $45 \mathrm{~K}$. pneumoniae isolates (44.4\%) were reported as nonsusceptible (intermediate and resistant) to ertapenem (Table 1). However, when the old 2009 breakpoints were used, ertapenem interpretation classified only $15(33.3 \%)$ of isolates as nonsusceptible and $30(66.7 \%)$ as susceptible. Of the 5 isolates that was counted as susceptible by the 2009 guidelines yet nonsusceptible by the new guidelines, 3 isolates were positive for the $b l a_{\mathrm{KPC}}$ gene; these isolates were susceptible to meropenem and imipenem. Among the isolates tested, $40.0 \%$ and $37.8 \%$ were nonsusceptible to imipenem and meropenem respectively at the new CLSI resistance breakpoint of $\geq 2 \mu \mathrm{g} /$ $\mathrm{mL}$ for both drugs (Table 1).

\begin{tabular}{|c|c|c|c|c|c|c|c|c|}
\hline \multirow[t]{3}{*}{ Antibiotic agent } & \multicolumn{4}{|c|}{ Older breakpoints $^{a}$} & \multicolumn{4}{|c|}{ Current breakpoints ${ }^{b}$} \\
\hline & \multicolumn{2}{|c|}{ Susceptible } & \multicolumn{2}{|c|}{ Nonsusceptible $^{c}$} & \multicolumn{2}{|c|}{ Susceptible } & \multicolumn{2}{|c|}{ Nonsusceptible $^{c}$} \\
\hline & No. & $\%$ & No. & $\%$ & No. & $\%$ & No. & $\%$ \\
\hline Imipenem & 34 & 75.6 & 11 & 24.4 & 27 & 60.0 & 18 & 40.0 \\
\hline Meropenem & 33 & 73.3 & 12 & 26.7 & 28 & 62.2 & 17 & 37.8 \\
\hline Ertapenem & 30 & 66.7 & 15 & 33.3 & 25 & 55.6 & 20 & 44.4 \\
\hline
\end{tabular}

${ }^{a}$ CLSI M100-S19 criteria [14,15]; ${ }^{b}$ CLSI M100-S22 criteria [16]; Intermediate and resistant. 


\begin{tabular}{lccc}
\hline $\begin{array}{l}\text { Table } 2 \text { Results of modified Hodge test (MHT) and polymerase chain reaction } \\
\text { (PCR) assay for bla } \boldsymbol{K}_{\text {KPC }} \text { gene on nonsusceptible Klebsiella pneumoniae isolates } \\
(\boldsymbol{n}=\mathbf{2 0})\end{array}$ & $\begin{array}{r}\text { Tolymerase chain reaction } \\
\text { results }\end{array}$ \\
\hline Modified Hodge test results & PCR+ve & PCR-ve & \\
& 14 & 3 & 17 \\
MHT+ve & 0 & 3 & 3 \\
MHT-ve & 14 & 6 & 20 \\
Total & & & \\
\hline
\end{tabular}

+ ve $=$ positive $;-v e=$ negative .

Real-time KPC PCR assay results were used to confirm that carbapenem resistance in $K$. pneumoniae isolates was due to production of a KPC. Of the 20 K. pneumoniae isolates with reduced susceptibility to ertapenem (defined as $\geq 1 \mu \mathrm{g} / \mathrm{mL}$ ), according to the revised clinical breakpoints, 14 isolates were found positive for KPCs by MHT and by PCR detection of the $b l_{\mathrm{KPC}}$ gene (Table 2). Of the remaining 6 isolates that were negative by PCR, 3 isolates were positive by MHT.

\section{Discussion}

The emergence and rapid spread of antibiotic-resistant $K$. pneumoniae isolates harbouring the $b l a_{\mathrm{KPC}}$ gene that encodes for carbapenemase production have complicated the management of patients' infections [1,2]. To our knowledge, this is the first published report of KPC-producing $K$. pneumoniae isolated from patients at a tertiary care hospital in Egypt. In our study we used ertapenem to screen for carbapenemases, as ertapenem is the least active carbapenem against KPCs [26] and as the use of this drug in automated or manual susceptibility testing has been found to be a highly sensitive method for the detection of KPCs $[26,27]$. Despite the limited number of isolates included, we were able to show a high prevalence of ertapenem non-susceptibility, accounting for $44.4 \%$ of $K$. pneumoniae isolates tested. This high prevalence reflected the new lower CLSI breakpoints for carbapenems. When the previous CLSI breakpoints for ertapenem were used (resistant $>4 \mu \mathrm{g} / \mathrm{mL}$; susceptible $\leq 2$ $\mu \mathrm{g} / \mathrm{mL}$ ), only $33.3 \%$ would be counted as nonsusceptible. A high prevalence of ertapenem resistance was similarly reported by many investigators in different countries $[5,19]$. For instance, in a study from China none of the 77 clinical isolates collected from 2002 to 2009 were susceptible to ertapenem and only $6.5 \%$ and $1.3 \%$ of isolates were susceptible to imipenem and meropenem respectively [28].

Of the 5 isolates that were counted as ertapenem-susceptible by the old CLSI M100-S19 breakpoints but nonsusceptible by the revised breakpoints, 3 isolates were positive for $b l a_{\mathrm{KPC}}$ genes and MHT, signifying the improved rate of detection of KPC-meditated resistance when using the new CLSI breakpoints. Likewise, the new breakpoints increased the proportion of isolates counted as nonsusceptible to imipenem and meropenem (to $40.0 \%$ and $37.8 \%$ respectively), although these were less than for ertapenem.

We also described in this study, a real-time PCR designed to detect and characterize genes encoding all KPC variants. Using this assay, we documented for the first time in Egypt the presence of isolates producing KPCs. Isolates with ertapenem MIC $\geq 1 \mu \mathrm{g} /$ $\mathrm{mL}$ were further investigated to determine the prevalence of KPC enzymes. We were able to confirm the presence of $b l a_{\text {KPC }}$ genes in $14(70.0 \%)$ of ertapenem-nonsusceptible isolates, which comprised $31.1 \%$ of all isolates tested, indicating that the increased prevalence of ertapenem non-susceptibility was predominantly attributed to KPCmediated resistance mechanisms in $K$. pneumoniae. Prevalence rates of KPCpositive K. pneumoniae isolates of $>30 \%$ have been recorded in some institutions in the eastern USA, in association with nosocomial outbreaks [27].

Our results suggest performing confirmatory testing for the presence of KPC for all ertapenem-resistant bacteria. All KPC-producing bacteria were also MHT positive, indicating the usefulness of doing this phenotypic testing. However, due to the more rapid turnaround time of PCR assays, this assay might be more suitable as an initial screening test for detecting KPCmediated carbapenem resistance. On the other hand, $\mathrm{PCR}$ is more technically challenging, prone to inhibition and may miss new variants of KPC arising from genetic mutation

Of the ertapenem-nonsusceptible isolates 6 were negative by real-time $b l_{\mathrm{KPC}}$ PCR and, of those, 3 isolates were positive by MHT. Two possibilities exist that may explain these 3 MHT-positive/KPC-PCR-negative isolates. First, as reported by Schechner et al. KPC PCR could be falsely negative due to inhibitory substances in the reaction or to technical inexperience of the laboratory [29]. However, the most probable reason could be the presence of other carbapenemases, such as the metallo-beta-lactamases and the member of the Serratia marcescens (SME) family of carbapenem-hydrolyzing beta-lactamases, SME-1, which can produce a positive result for MHT but negative for $b l_{\mathrm{KPC}}$. So although the new CLSI recommendations lowered the breakpoints of carbapenems and removed the requirement for testing for carbapenemase (e.g. MHT) to determine susceptibility [15], performing MHT as an adjunct to KPC PCR may increase the likelihood of detecting other carbapenemases. Furthermore, the current recommendation is to still 
to perform MHT for infection control and epidemiological purposes.

Our study had some limitations. First, the number of isolates included in the study was limited by the low incidence of $K$. pneumoniae-associated infections in our institution during the study; nonetheless, the available results provided robust pilot data. Secondly, molecular detection of bla ${ }_{\mathrm{KPC}}$ genes was further limited to isolates nonsusceptible to ertapenem. However, this did not substantially compromise our study findings, especially when using the new lower CLSI breakpoints for interpretation. Thirdly, we did not screen our isolates for other resistance determinants, such as AmpC or outer membrane proteins, owing to limited funding available.

In summary, our data showing an increased prevalence of ertapenemnonsusceptible $K$. pneumoniae isolates partly reflects lowering of clinical breakpoints but also indicates the spread of carbapenemases, principally KPC types, in Suez Canal University hospital, Egypt. Confirmatory testing for the presence of KPCs is required for all ertapenem-resistant bacteria. Real-time PCR assay described here provides a useful tool to rapidly and accurately detect bla ${ }_{\mathrm{KPC}}$-positive bacteria, which is an important step in controlling their spread.

\section{Acknowledgements}

Funding: No specific funding was received for this study.

Competing interests: None declared.

\section{References}

1. Nordmann P, Cuzon G, Naas T. The real threat of Klebsiella pneumoniae carbapenemase-producing bacteria. Lancet Infectious Diseases, 2009, 9:228-236.

2. Arnold RS et al. Emergence of Klebsiella pneumoniae carbapenemase-producing bacteria. Southern Medical Journal, 2011, 104:40-45.

3. Yigit Het al. Novel carbapenem-hydrolyzing beta-lactamase, KPC-1, from a carbapenem-resistant strain of Klebsiella pneumoniae. Antimicrobial Agents and Chemotherapy, 2001, 45:11511161.

4. Hirsch EB et al. Emergence of KPC-producing Klebsiella pneumoniae in Texas. Diagnostic Microbiology and Infectious Disease, 2011, 69:234-235.

5. Centers for Disease Control and Prevention (CDC). Carbapenem-resistant Klebsiella pneumoniae associated with a longterm-care facility-West Virginia, 2009-2011. Morbidity and Mortality Weekly Report, 2011, 60:1418-1420.

6. Bratu S et al. Rapid spread of carbapenem-resistant Klebsiella pneumoniae in New York city: a new threat to our antibiotic armamentarium. Archives of Internal Medicine, 2005, 165:14301435.

7. Brandon Kitchel et al. Molecular epidemiology of KPC-producing Klebsiella pneumoniae isolates in the United States: clonal expansion of multilocus sequence type 258. Antimicrobial Agents and Chemotherapy, 2009, 53:3365-3370.

8. Steinmann Jet al. Outbreak due to a Klebsiella pneumoniae strain harbouring KPC-2 and VIM-1 in a German university hospital, July 2010 to January 2011. Eurosurveillance, 2011, 16:19944.

9. Chung KP et al. Arrival of Klebsiella pneumoniae carbapenemase (KPC)-2 in Taiwan. Journal of Antimicrobial Chemotherapy, 2011, 66:1182-1184.

10. Beirao EM et al. Clinical and microbiological characterization of KPC-producing Klebsiella pneumoniae infections in Brazil. Brazilian Journal of Infectious Diseases, 2011, 15:69-73.

11. Gomez-Gil MRet al. Detection of KPC-2-producing Citrobacter freundii isolates in Spain. Journal of Antimicrobial Chemotherapy, 2010, 65:2695-2697.

12. Leavitt A et al. Molecular epidemiology, sequence types, and plasmid analyses of KPC-producing Klebsiella pneumoniae strains in Israel. Antimicrobial Agents and Chemotherapy, 2010, 54:3002-3006.

13. Souli Met al. An outbreak of infection due to beta-lactamase Klebsiella pneumoniae carbapenemase 2-producing K. pneu- moniae in a Greek university hospital: molecular characterization, epidemiology, and outcomes. Clinical Infectious Diseases, 2010, 50:364-373.

14. Performance standards for antimicrobial susceptibility testing: 19th informational supplement. CLSI document M100-S19. Wayne, Pennsylvania, Clinical and Laboratory Standards Institute, 2009.

15. Performance standards for antimicrobial susceptibility testing, 20th informational supplement: M100-S20 \& M100-S-20-U. Wayne, Pennsylvania, Clinical and Laboratory Standards Institute, 2010.

16. Performance standards for antimicrobial susceptibility testing; 22nd informational supplement. M100-S22. Wayne, Pennsylvania, Clinical and Laboratory Standards Institute, 2012.

17. Endimiani A et al. Presence of plasmid-mediated quinolone resistance in Klebsiella pneumoniae isolates possessing bla $a_{\mathrm{KPC}}$ in the United States. Antimicrobial Agents and Chemotherapy, 2008, 52:2680-2682.

18. Neuner EA et al. Treatment and outcomes in carbapenemresistant Klebsiella pneumoniae bloodstream infections. Diagnostic Microbiology and Infectious Disease, 2011, 69:357-362.

19. Pfeifer Y, Cullik A, Witte W. Resistance to cephalosporins and carbapenems in Gram-negative bacterial pathogens. International Journal of Medical Microbiology, 2010, 300:371-379.

20. Doumith $M$ et al. Molecular mechanisms disrupting porin expression in ertapenem-resistant Klebsiella and Enterobacter spp. clinical isolates from the UK. Journal of Antimicrobial Chemotherapy, 2009, 63:659-667.

21. Cuzon G et al. In vivo selection of imipenem-resistant Klebsiella pneumoniae producing extended-spectrum beta-lactamase CTX-M-15 and plasmid-encoded DHA-1 cephalosporinase. International Journal of Antimicrobial Agents, 2010, 35:265-268.

22. Raghunathan A, Samuel L, Tibbetts RJ. Evaluation of a real-time PCR assay for the detection of the Klebsiella pneumoniae carbapenemase genes in microbiological samples in comparison with the modified Hodge test. American Journal of Clinical Pathology, 2011, 135:566-571

23. Hindiyeh $\mathrm{M}$ et al. Rapid detection of blaKPC carbapenemase genes by internally controlled real-time PCR assay using Bactec blood culture bottles. Journal of Clinical Microbiology, 2011, 49(7):2480-2484.

24. Doern CD, Dunne WM Jr, Burnham CA. Detection of Klebsiella pneumoniae carbapenemase (KPC) production in non-Kleb- 
siella pneumoniae Enterobacteriaceae isolates by use of the Phoenix, Vitek 2, and disk diffusion methods. Journal of Clinical Microbiology, 2011, 49:1143-1147.

25. Carvalhaes CG et al. Cloverleaf test (modified Hodge test) for detecting carbapenemase production in Klebsiella pneumoniae: be aware of false positive results. Journal of Antimicrobial Chemotherapy, 2010, 65:249-251.

26. Landman D et al. Accuracy of carbapenem nonsusceptibility for identification of KPC-possessing Enterobacteriaceae by use of the revised CLSI breakpoints. Journal of Clinical Microbiology, 2011, 49:3931-3933.

27. Endimiani $\mathrm{A}$ et al. Evaluation of updated interpretative criteria for categorizing Klebsiella pneumoniae with reduced carbap- enem susceptibility. Journal of Clinical Microbiology, 2010, 48:4417-4425.

28. Hu $\mathrm{F}$ et al. Emergence of carbapenem-resistant clinical Enterobacteriaceae isolates from a teaching hospital in Shanghai, China. Journal of Medical Microbiology, 2012, 61:132-136.

29. Schechner $V$ et al. Evaluation of PCR-based testing for surveillance of KPC-producing carbapenem-resistant members of the Enterobacteriaceae family. Journal of Clinical Microbiology, 2009, 47:3261-3265. 